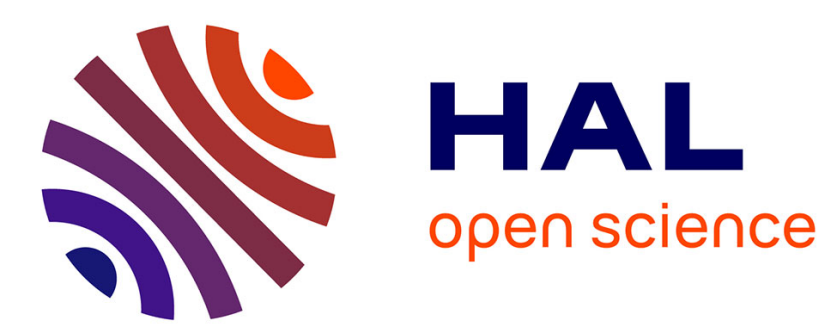

\title{
Interplay between spin-glass clusters and geometrical frustration
}

Fabio Zimmer, Cintia Silva, Sergio Magalhaes, Claudine Lacroix

\section{To cite this version:}

Fabio Zimmer, Cintia Silva, Sergio Magalhaes, Claudine Lacroix. Interplay between spin-glass clusters and geometrical frustration. Physical Review E : Statistical, Nonlinear, and Soft Matter Physics, 2014, 89 (2), pp.02210. 10.1103/PhysRevE.89.022120 . hal-00949042

\section{HAL Id: hal-00949042 \\ https://hal.science/hal-00949042}

Submitted on 26 Feb 2014

HAL is a multi-disciplinary open access archive for the deposit and dissemination of scientific research documents, whether they are published or not. The documents may come from teaching and research institutions in France or abroad, or from public or private research centers.
L'archive ouverte pluridisciplinaire HAL, est destinée au dépôt et à la diffusion de documents scientifiques de niveau recherche, publiés ou non, émanant des établissements d'enseignement et de recherche français ou étrangers, des laboratoires publics ou privés. 


\title{
Interplay between Spin-Glass clusters and frustration
}

\author{
F. M. Zimmer, C. F. Silva, S. G. Magalhaes, C. Lacroix \\ Dep. de Fisica, Universidade Federal de Santa Maria, 97105-900, Santa Maria, RS, Brazil \\ Instituto de Fisica, Universidade Federal Fluminense, 24210-346 Niteroi, RJ, Brazil and \\ Institut Neel, CNRS/UJF, 25 avenue des Martyrs, BP 166, F-38042 Grenoble, Cedex 09, France
}

\begin{abstract}
The presence of spin glass (SG) order in highly geometrically frustrated systems is analyzed with a cluster SG model. The model considers infinite-range disordered interactions among cluster magnetic moments and the $J_{1}-J_{2}$ model couplings between Ising spins of the same cluster. This model can introduce two source of frustration: one coming from the disordered interactions and other coming from the $J_{1}-J_{2}$ intracluster interactions (intrinsic frustration). The framework of one-step replica symmetry breaking is adopted to obtain an one-cluster problem that is solved exactly. As mean results are build phase diagrams of the temperature $T$ versus intensity of the disorder $J$, where the paramagnetic/SG phase transition occurs at $T_{f}$ when $T$ decreases for high $J$ values. For low $J$ values, the SG order is absent for antiferromagnetic clusters without intrinsic frustration. However, the SG can be observed within the intracluster intrinsic frustration regime even for lower intensity of disorder. Particularly, the results indicate that the presence of small clusters in geometrically frustrated antiferromagnetic systems can help to stabilize the SG order within a weak disorder.
\end{abstract}

\section{INTRODUCTION}

The interplay between geometrical frustration (GF) and disorder is an interesting problem with many open questions. For instance, there are several geometrically frustrated magnets that exhibit spin glass (SG)like behavior as, $\mathrm{Y}_{2} \mathrm{Mo}_{2} \mathrm{O}_{7},{ }^{4,5} \mathrm{Zn}_{1-x} \mathrm{Cd}_{x} \mathrm{Cr}_{2} \mathrm{O}_{4},{ }^{6}$ and $\mathrm{ZnCr}_{2(1-x)} \mathrm{Ga}_{2 x} \mathrm{O}_{4} \cdot{ }^{7,8}$ Nevertheless, the level of disorder presented in some of these geometrically frustrated systems has been estimated to be extremely small. ${ }^{4,7}$ In fact, the amount of disorder to stabilize a glassy state in these real systems is much less than the predicted from usual mean field theory for SG. ${ }^{5,9}$ Therefore, a very intriguing issue is how a SG-like state is stabilized in these geometrically frustrated systems with very small disorder. This issue is the main subject of the present work.

It should be remarked that there are few theoretical works to account the interplay between disorder and GF in systems presenting SG-like state (see, for instance, Refs. 3 and 10). Nevertheless, these studies do not consider clusters. Therefore, new approaches must be required in order to understand the low-disorder SG phase in highly geometrically frustrated systems in which, for instance, magnetically correlated spin clusters can be present as in the case of $\mathrm{ZnCr}_{2(1-x)} \mathrm{Ga}_{2 x} \mathrm{O}_{4}{ }^{8}$.

In this work we present a new approach in which the presence of spin clusters can be an essential element to account for the extremely large sensitivity to the effects of disorder in geometrically frustrated systems. We consider clusters of spins with non-disordered intracluster spin interactions. The disorder appears only as a quenched random magnetic interaction between these spin clusters. For an appropriated choice of intracluster interactions, GF can arise leading to a degeneracy of intracluster spins configurations. In that case, the intercluster random interaction could select from the manifold of spins configurations those ones which not only avoid, for instance, the fully compensation of the total cluster magnetic moment but also enhance hugely the spin cluster sensitivity to the intercluster random interaction stabilizing a cluster SG order even when the intercluster random interaction is very weak.

Motivated by the above considerations, we study a simple cluster spin model in which it is possible to introduce intracluster frustration and disorder. This model has two types of interactions: intercluster long-range disordered interactions and intracluster short-range interactions between first $\left(J_{1}\right)$ and second neighbors $\left(J_{2}\right)$ which can be a $\mathrm{AF}$ interaction or a ferromagnetic (FE) one. The shortrange interactions are between Ising spins on a square lattice that belong to the same cluster. Therefore, the intracluster interaction is given by the so called $J_{1}-J_{2}$ model that can introduce frustration by adjusting the relation between $J_{1}$ and $J_{2}{ }^{2}$ This we call intrinsic frustration.

\section{GENERAL FORMULATION}

The model can be obtained from the Ising spin lattice that is divided into $N_{c l}$ clusters with $n_{s}$ sites in each cluster. ${ }^{16}$ In the present approach the intercluster interactions are assumed infinite-range and disordered, while the intracluster interactions are short-range. ${ }^{16}$ This model can be described by the Hamiltonian

$$
H=-\sum_{\nu \lambda}^{N_{c l}} J_{\nu \lambda} S_{\nu} S_{\lambda}-\sum_{\nu}^{N_{c l}} \sum_{(i, j)}^{n_{s}} J_{i j}^{\nu} s_{i \nu} s_{j \nu}
$$

where $J_{\nu \lambda}$ is a random variable that follows a gaussian distribution with mean zero and variance $J^{2} / N n_{s}$ $\left(N=N_{c l} n_{s}\right)$. In Eq. (1), $J_{\nu \lambda}$ couples all distinct pairs of clusters, in which $S_{\nu}=\sum_{i}^{n_{s}} s_{i \nu}$ is the magnetic moment of cluster $\nu$ with $s_{i \nu}$ representing the Ising spin of site $i$ of cluster $\nu$. The intracluster interaction follows the $J_{1}-J_{2}$ 
model coupling written as

$$
\sum_{(i, j)}^{n_{s}} J_{i, j}^{\nu} s_{i \nu} s_{j \nu}=\sum_{(i, j)_{1}}^{n_{s}} J_{1} s_{i \nu} s_{j \nu}+\sum_{(i, j)_{2}}^{n_{s}} J_{2} s_{i \nu} s_{j \nu}
$$

where the sum $(i, j)_{1}\left((i, j)_{2}\right)$ runs over all nearestneighbor (next-nearest-neighbor) sites of cluster $\nu$ with a square lattice geometry. In this work is studied two types of couplings between nearest-neighbor $J_{1}$ and nextnearest-neighbor $J_{2}$. The first one considers $J_{1}$ and $J_{2}$ antiferromagnetic (AF), while in the second $J_{1}$ is ferromagnetic (FE) and $J_{2} \mathrm{AF}$.

The replica method is used to carry out the disorder average. The averaged free energy per cluster is $\beta f=\lim _{n \rightarrow 0}\left(\left\langle Z^{n}\right\rangle_{J_{\nu \lambda}}-1\right) / N n$, where $\langle\ldots\rangle_{J_{\nu \lambda}}$ means the average over the quenched disorder of $J_{\nu \lambda}$ and $Z^{n}$ is the replicated partition function. This produces an effective replica Hamiltonian

$H=-\frac{\beta J^{2}}{2 N_{c l} n_{s}} \sum_{\nu \lambda}^{N_{c l}} \sum_{\alpha, \gamma}^{n} S_{\nu}^{\alpha} S_{\nu}^{\gamma} S_{\lambda}^{\alpha} S_{\lambda}^{\gamma}-\sum_{\nu}^{N_{c l}} \sum_{\alpha}^{n} \sum_{(i, j)}^{n_{s}} J_{i, j}^{\nu} s_{i \nu}^{\alpha} s_{j \nu}^{\alpha}$

where $\beta=1 / T$ ( $T$ is the temperature), and $\alpha$ and $\gamma$ are replica labels.

The four-spin cluster term is decoupled introducing the replica matrix elements $\{Q\}$ via Hubbard-Stratonovitch transformations. The free energy per cluster is then obtained as

$$
\begin{array}{r}
\frac{\beta f}{N_{c l}}=\lim _{n \rightarrow 0} \frac{1}{n}\left\{\frac{\beta^{2} J^{2}}{2 n_{s}^{2}} \sum_{\alpha \gamma} Q_{\alpha \gamma}^{2}-\frac{1}{N_{c l}} \ln \operatorname{Tr} \exp \right. \\
\left.\left[\beta \sum_{\nu}\left(\sum_{\alpha} \sum_{(i, j)}^{n_{s}} J_{i, j}^{\nu} s_{i \nu}^{\alpha} s_{j \nu}^{\alpha}+\sum_{\alpha \gamma} \frac{\beta J^{2}}{n_{s}^{2}} Q_{\alpha \gamma} S_{\nu}^{\alpha} S_{\nu}^{\gamma}\right)\right]\right\}
\end{array}
$$

where the saddle-point equations are used resulting in $Q^{\alpha \gamma}=\frac{1}{N_{c l}}<\sum_{\nu} S_{\nu}^{\alpha} S_{\nu}^{\gamma}>$.

The Parisi's scheme of $1 \mathrm{~S}-\mathrm{RSB}^{11}$ is adopted to parametrize the replica matrix as $R=Q_{\alpha \alpha}$ and

$$
Q_{\alpha, \gamma}= \begin{cases}Q_{1} & \text { if } I(\alpha / a)=I(\gamma / a) \\ Q_{0} & \text { if } I(\alpha / a) \neq I(\gamma / a)\end{cases}
$$

where $I(x)$ gives the smallest integer which is greater than or equal to $x$. The order parameter $R=<S_{\nu}^{\alpha} S_{\nu}^{\alpha}>$ represents the replica diagonal correlation of the cluster magnetic moment. Different from the canonical Ising SG models (where $R=1$ ), here it can range from 0 to $n_{s}^{2}$. $R$ can be interpreted as the intensity of the cluster magnetic moment. ${ }^{16}$ Particularly, $R$ is strongly affected by AF intracluster interactions that can introduce compensated magnetic moments decreasing the intensity of the cluster magnetic moment $(R \rightarrow 0)$. The replica symmetry breaking is given by $Q_{1}-Q_{0}$, which is the SG order parameter. The parameter $a$ represents the size of diagonal blocks of the 1S-RSB solution.
In this approximation, the free energy is obtained as

$$
\begin{array}{r}
\frac{\beta f}{N_{c l}}=\frac{\beta^{2} J^{2}}{4 n_{s}^{2}}\left[R^{2}+a\left(Q_{1}^{2}-Q_{0}^{2}\right)-Q_{1}^{2}\right] \\
-\frac{1}{a} \int D z \ln \int D v[K(z, v)]^{a},
\end{array}
$$

where $K(z, v)=\int D \xi \operatorname{Tr} e^{-\beta H_{e f}}, \quad \int D x=$ $\int_{-\infty}^{\infty} d x \frac{e^{x^{2} / 2}}{\sqrt{2 \pi}}(x=z, v$ or $\xi)$ and

$$
H_{e f}=-\sum_{(i, j)_{1}}^{n_{s}} J_{1} s_{i \nu} s_{j \nu}-\sum_{(i, j)_{2}}^{n_{s}} J_{2} s_{i \nu} s_{j \nu}-h S_{\nu}
$$

represents the effective one-cluster model with the 1SRSB self-consistent field

$$
h=\frac{J}{n_{s}}\left[\sqrt{Q_{1}-Q_{0}} v+\sqrt{R-Q_{1}} \xi+\sqrt{Q_{0}} z\right] .
$$

The parameters $Q_{1}, Q_{0}, R$ and $a$ are obtained from the extreme of the free energy (6). The magnetic cluster susceptibility $\chi$ and specific heat $c_{v}$ are derived from Eq. (6): $\chi=\beta\left(R-Q_{1}+a\left(Q_{1}-Q_{0}\right)\right)$ and $c_{v}=-\frac{\partial U}{\partial T}$ where $U=-T^{2} \frac{\partial(\beta F)}{\partial T}$.

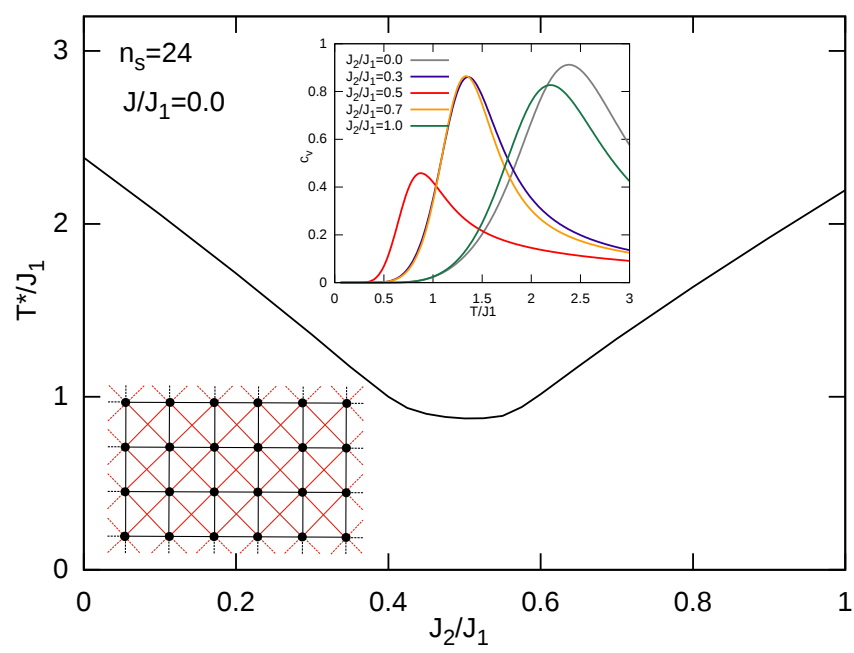

FIG. 1: Temperature of maximum value of the specific heat $c_{v}, T^{*}$, versus $J_{2} / J_{1}$ for an AF finite-size system with $n_{s}=24$ and $J=0$ (without disorder). The lattice geometry is showed at left-bottom panel where is considered periodic boundary conditions. Inset exhibits the $c_{v}$ behavior for several $J_{2} / J_{1}$ values. The minimum of $T^{*}$ appears at $J_{2} / J_{1}=0.5$ in the region of stronger intrinsic frustration.

\section{RESULTS AND DISCUSSION}

The effective one-cluster problem (Eqs. (6)-(8)) is now numerically solved by using exact diagonalization. We consider clusters with $n_{s}$ Ising spins on a bidimensional lattice with short-range interactions with intensities $J_{1}$ 

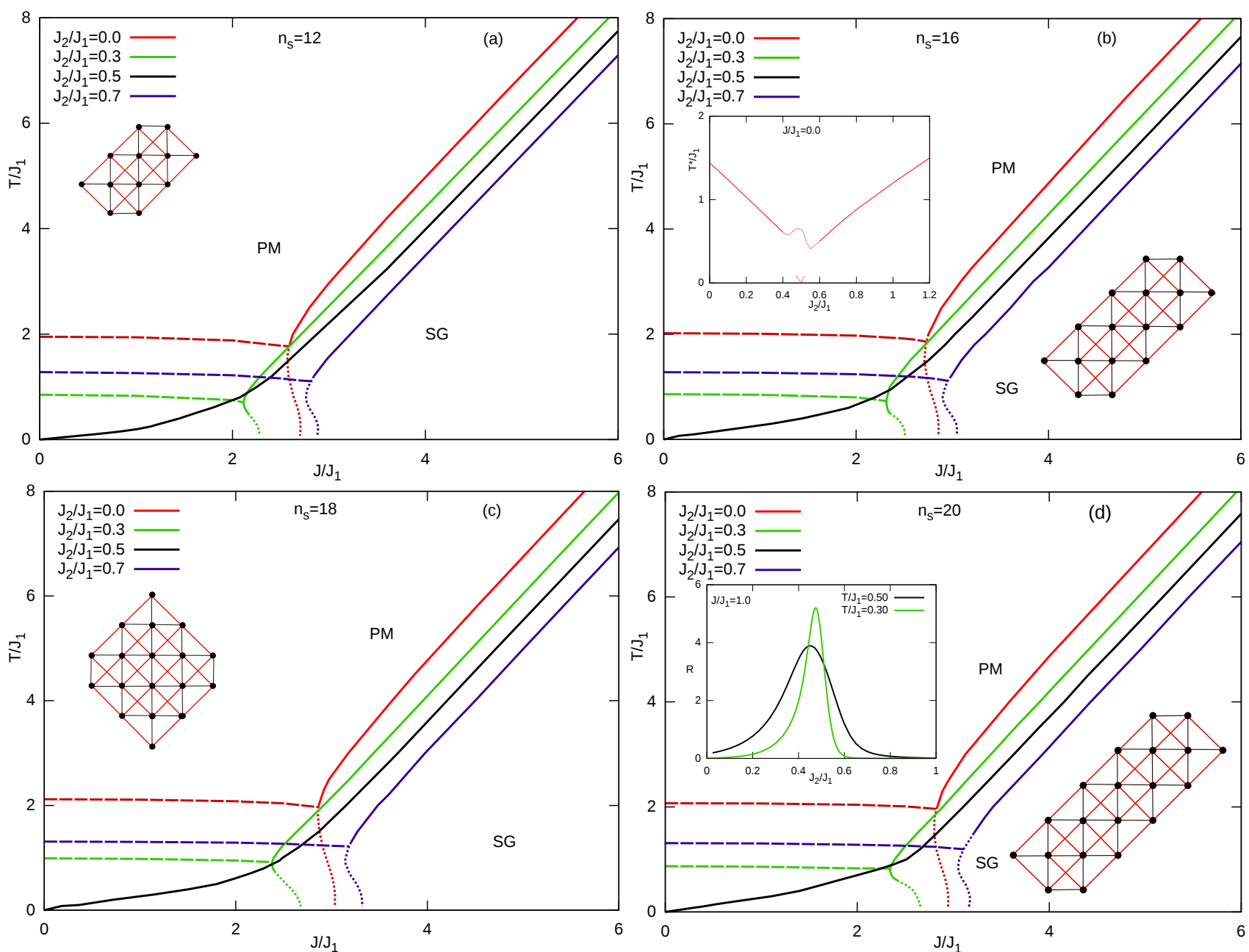

FIG. 2: Phase diagrams $T / J_{1}$ versus $J / J_{1}$ for several values of $J_{2} / J_{1}$ with different cluster sizes and antiferromagnetic intracluster couplings. Cluster shapes without periodic boundary conditions are presented in each panel, where black and red lines represent first and second neighbors interactions respectively. Solid and dotted lines represent PM/SG second and first-order transitions, respectively. Dashed lines correspond to the maximum of the susceptibility (discussed in Fig. (4)). Inset in (b) shows the $T^{*} / J_{1}$ vs $J_{2} / J_{1}$ behavior for $J=0$. Inset in (d) shows the order parameter $R$ as a function of $J_{2} / J_{1}$ within the PM phase at low $T\left(J / J_{1}=1.0\right.$ and $T / J_{1}=0.3$ and 0.5$)$. The SG phase is always found when the disorder increases for AF Ising cluster, but it is stabilized at lower intensities of disorder in the presence of intracluster frutration.

and $J_{2}$. The analyses are done for two different intracluster $J_{1}-J_{2}$ couplings: one with both $J_{1}$ and $J_{2}$ antiferromagnetic (section III A) and other with $J_{1}$ ferromagnetic and $J_{2}$ antiferromagnetic (section III B). Particularly, the intercluster disorder $J$ results from the 1S-RSB approximation for the effective field $h$ (Eq. 8). Therefore, the SG order is characterized by the RSB solution $\left(Q_{1}-Q_{0}>0\right)$. It is also important to point that $J$ is divided by $n_{s}$ in Eq. (8), in which $J$ is related to the intensity of the disorder per spin.

\section{A. Antiferromagnetic intracluster interactions}

First, we present results for the classical antiferromagnetic $J_{1}-J_{2}$ model in a finite-size system without inter- cluster disorder (decoupled clusters with $J / J_{1}=0$ ). For example, the specific heat $c_{v}$ for a square lattice with 24 spins and periodic boundary conditions (PBC) is showed in the inset of Fig. (1). The $c_{v}$ curve displays a maximum value at temperature $T^{*} . T^{*}$ for different ratios $J_{2} / J_{1}$ (parameter that controls the intrinsic frustration) is used to build the phase diagram of Fig. (1), which presents the behavior expected from this model. ${ }^{13-15}$ At temperatures below $T^{*}$, the finite-cluster results suggest that the Neel and collinear (lines of spins parallel are coupled antiparallel) antiferromagnetic like orders are found at lower and higher values of $J_{2} / J_{1}$, respectively. ${ }^{13}$ It is important to observe that $T^{*}$ is minimum at $J_{2} / J_{1}=0.5$, in which the maximum intrinsic frustration occurs.

The effects of disorder are introduced by considering the intercluster disorder $J \neq 0$ for different cluster sizes 
with even numbers of spins and without PBC. For instance, Fig. 2 shows that $J$ is able to introduce the SG phase, where a second-order transition (solid lines) from the paramagnetic (PM) phase to the SG order occurs when the temperature decreases for high $J$ values. As $J$ diminishes, the transition becomes first-order (dotted lines), in which a reentrance can appear for $J_{2}=0$ (no intracluster frustration). This reentrance is associated with the interesting phenomena of inverse freezing (IF) that was already pointed in Ref. 12 , in which $J_{2}=0$. Here, the phase diagrams exhibited in Fig. (2) with small $J_{2} / J_{1}$ values are qualitatively the same as those ones of Ref. 12. The IF arises as a consequence of the interplay between intercluster disorder and short-range AF interactions. The intercluster disorder favors the SG phase that can present a higher entropic content than a lowtemperature cluster PM phase. This PM phase appears when the AF interaction is large, in which the intracluster spins become AF compensated establishing clusters with low magnetic moments without long-range order.

However, $J_{2}$ can introduce intracluster frustration effects on $T_{f}$. For instance, the maximum intracluster intrinsic frustration appears at $J_{2} / J_{1}=0.5$ for clusters with PBC (see Fig. (1)), where the number of intracluster $J_{1}$ and $J_{2}$ interactions are the same. Nevertheless, the $\mathrm{PBC}$ are not used for the disordered intercluster problem. Instead of this the cluster shapes of Fig. (2) are chosen such that the number of intracluster interactions between nearest-neighbors and next-near-neighbors are very close (see red and black lines in the insets of Fig. 2) in order to explore the property of intrinsic frustration for $J_{2} / J_{1}$ very close to 0.5 . In this case, the $T^{*}$ dependence on $J_{2} / J_{1}$ for $J=0$ (without disorder) exhibits a similar behavior as that one of Fig. (1) (see inset of Fig. $2-\mathrm{b})$. However, the $T^{*}$ is displaced to lower temperatures and its minimum at $J_{2} / J_{1}=0.5$ is hard to be located because of the finite size of the cluster. ${ }^{13}$ Nevertheless, the more important effect occurs when $J>0$, in which the $T_{f}$ behavior for $J_{2} / J_{1}$ near to the intracluster frustrated regime can be analyzed. For instance, $T_{f}$ appears at lower temperatures when $J_{2}$ increases, but the more relevant result is obtained for $J_{2} / J_{1}=0.5$, where the SG phase occurs at lower intensities of disorder. This result seems to be independent of the cluster size. It means that the intracluster intrinsic frustration favors the SG order, which appears at low disordered regimes.

To analyze this point, the inset of Fig. (2-d) exhibits the correlation $R$ as a function of $J_{2} / J_{1}$ for low temperatures in the PM region close to the PM/SG phase transition. The intracluster frustration causes an increment in the $R$ curves, which directly impacts on the intensity of the total cluster magnetic moment. This can contribute to the intercluster long-range disordered interactions at the same time that prevents the occurrence of the PM phase with small cluster magnetic moment. To put in another way, the cluster magnetic moment is maximized in the presence of intrinsic frustration and therefore the sensitivity to intercluster disordered interactions is en- hanced.

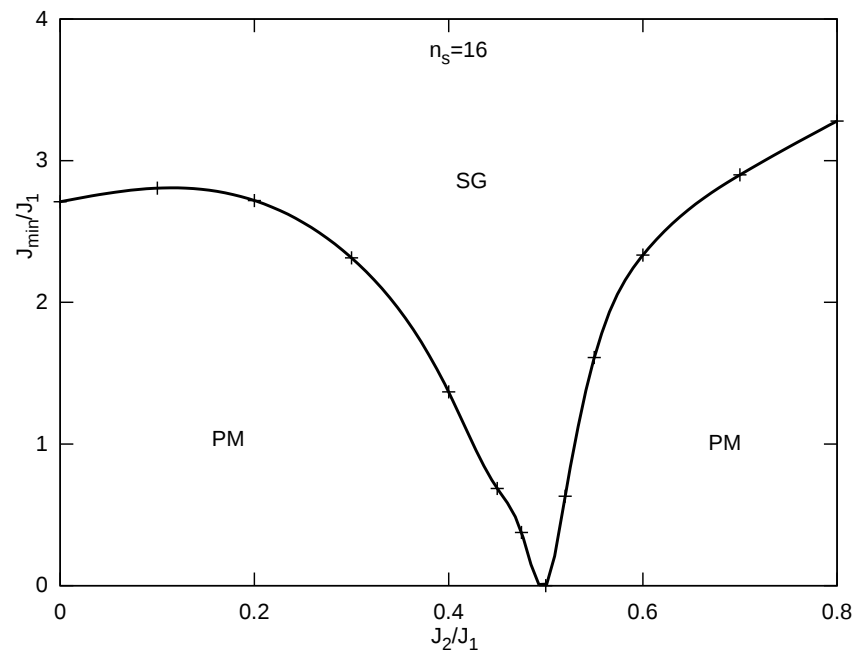

FIG. 3: Phase diagram $J_{\min } / J_{1}$ versus $J_{2} / J_{1}$ for $n_{s}=16$, in which $J_{\min }$ is the minimum intensity of intercluster disorder able to generate the SG phase with AF cluster. The cluster shape is the same as Fig. (2-b).

The effects of $J_{2} / J_{1}$ on the intercluster disorder can also be analyzed in Fig. (3) that exhibits the minimal intensity of disorder $J_{\min }$ required to get the SG phase, as a function of $J_{2} / J_{1}$. This phase diagram shows that the SG can always be obtained for a certain range of temperature when $J>J_{\min }$, while the PM phase is stable in the whole range of temperature for $J<J_{\min }$. The intensity of this minimum disorder is clearly diminishing as the intrinsic frustration increases. For instance, the SG phase can be obtained for extremely small $J$ as $J_{2} / J_{1} \rightarrow 0.5$. In other words, the intrinsic frustration potentiates the intercluster disordered couplings favoring the cluster SG phase.

Figure 4 exhibits the 1S-RSB order parameters for different rations of $J_{2} / J_{1}$. It enforces the previous discussion related with the intensity of the cluster magnetic moment $(R)$, which depends on the temperature, $J$ and $J_{2}$. For $J_{2}=0, R$ goes to zero as the temperature decreases for small $J$ values. It indicates that the cluster spins become AF compensated affecting the intercluster coupling, which remains in the PM phase down to zero temperature. On the other side, the intracluster intrinsic frustration can bring a new scenario, in which degenerate cluster spin configurations with uncompensated spins are thermodinamically favored. It can reflect on the increment of the magnetic moment of clusters as compared to other $J_{2} / J_{1}$ regimes. This increment of $R$ favors the intercluster coupling that can generate the $S G$ phase at lower disordered regimes ( $\operatorname{see} Q_{1}-Q_{0}>0$ for $J_{2} / J_{1}=0.5$ in Fig. (4)).

The cluster magnetic susceptibility $\chi$ can also be explored to clarify the low disorder regime (see inset of Fig. 4). $\chi$ shows a Curie-Weiss like behavior at high temperature cluster PM phase. However, as previously 


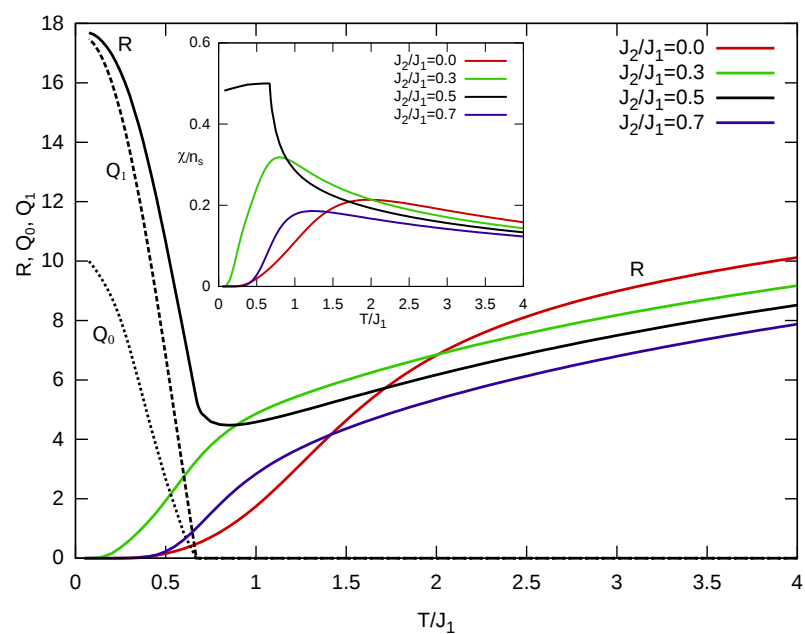

FIG. 4: Order parameters showed for a low disordered regime $\left(J / J_{1}=2.0\right)$, several $J_{2} / J_{1}$ values and $n_{s}=16$ with the same AF cluster shape as Fig. (2-b). Inset exhibits the susceptibility as a function of the temperature. The intrinsic frustration can increase the intensity of the magnetic moment of clusters $R$ favoring the occurrence of cluster SG phase at lower disorder.

discussed, one can found a low temperature PM phase, in which $\chi$ decreased by diminishing $T$ from a certain value $T_{\chi}^{*}$ (temperature of the smooth maximum in $\chi$ ). In this case, low magnetic moment clusters characterize the PM phase that arises as a consequence of the strong shortrange $\mathrm{AF}$ interactions without long-range order. The location of $T_{\chi}^{*}$ in the phase diagrams of Fig. (2) is given by the dashed lines, that show clearly the dependence of $T_{\chi}^{*}$ on the short-range interactions. Particularly, the intracluster frustration can eliminate the low temperature PM and the system presents SG with the susceptibility weakly dependent on temperature (see $\chi$ for $J_{2} / J_{1}=0.5$ in Fig. (4)).

\section{B. Ferromagnetic and Antiferromagnetic intracluster interactions}

Now, ferromagnetic and antiferromagnetic intracluster interactions are considered between first and second neighbors, respectively. For ferromagnetic clusters $\left(J_{2} / J_{1}<0.5\right)$, the phase diagrams exhibit the SG order even for lower values of $J / J_{1}$ (see Fig. (5-a)). When $J_{2} / J_{1}$ increases $\left(J_{2} / J_{1}>0.5\right)$, the antiferromagnetic short-range intracluster interactions become stronger and the SG phase is only observed for higher intensities of disorder. Furthermore, a reentrant first-order PM/SG transition appears. Particularly, at $J_{2} / J_{1}$ very close to 0.5 (see inset of Fig. (5-a)), the intracluster intrinsic frustration occurs and the SG phase is found at $J \rightarrow 0$. As in the case of $\mathrm{AF} J_{1}-J_{2}$ interactions, the results for other cluster sizes explored $\left(n_{s}=12,18\right.$ and 20) are qualitatively the same.

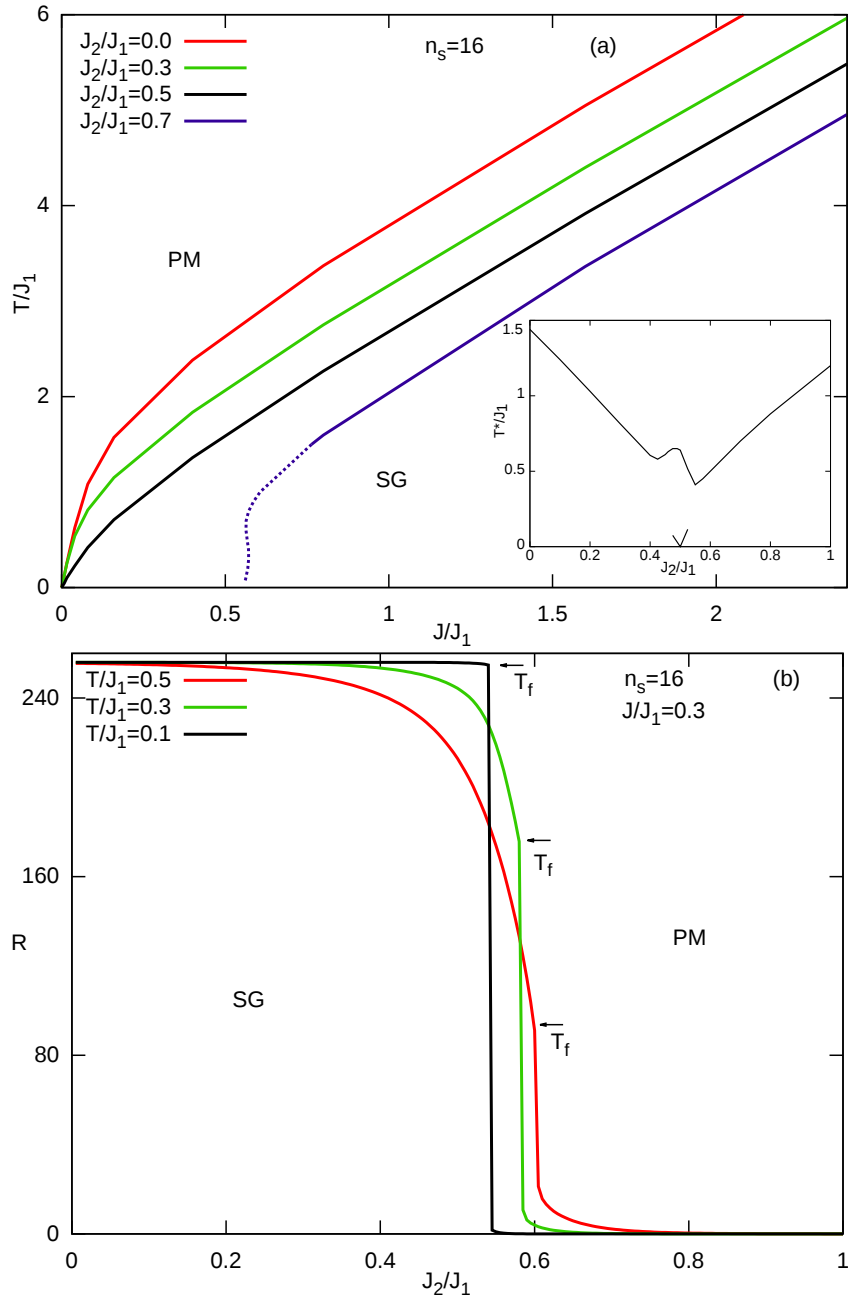

FIG. 5: Results for FE and AF intracluster interactions with $n_{s}=16$ and the same cluster shape as Fig. (2-b). (a) Phase diagrams $T / J_{1}$ versus $J / J_{1}$ for several values of $J_{2} / J_{1}$. (b) Order parameter $R$ as a function of $J_{2} / J_{1}$ for lower temperatures. Inset exhibits the behavior of $T^{*} / J_{1}$ as a function of $J_{2} / J_{1}$ for $J=0$.

The behavior of $R$ in Fig. (5-b) shows two different intracluster regimes: one for lower $J_{2} / J_{1}$ (where $R$ is maximum) and other for higher $J_{2} / J_{1}$ ( $R$ is minimum). It can help to explain the presence of the cluster SG phase for lower $J$ when the ferromagnetic intracluster interactions are dominant. In this case, the intercluster disordered couplings are enhanced by the large cluster magnetic moments. On the other hand, intracluster AF compensation makes the clusters less propense to the intercluster disordered interactions. Therefore, the SG phase can not be found at lower intensities of $J$ when $J_{2}$ is higher. Fig. (6) also enforces this behavior, in which FE clusters are able to present the cluster SG order within a very small disordered regime, while AF clusters require higher disorder to show the SG phase. In addition, the effects of intrinsic frustration are less pronounced than in the case analysed in section III A. 


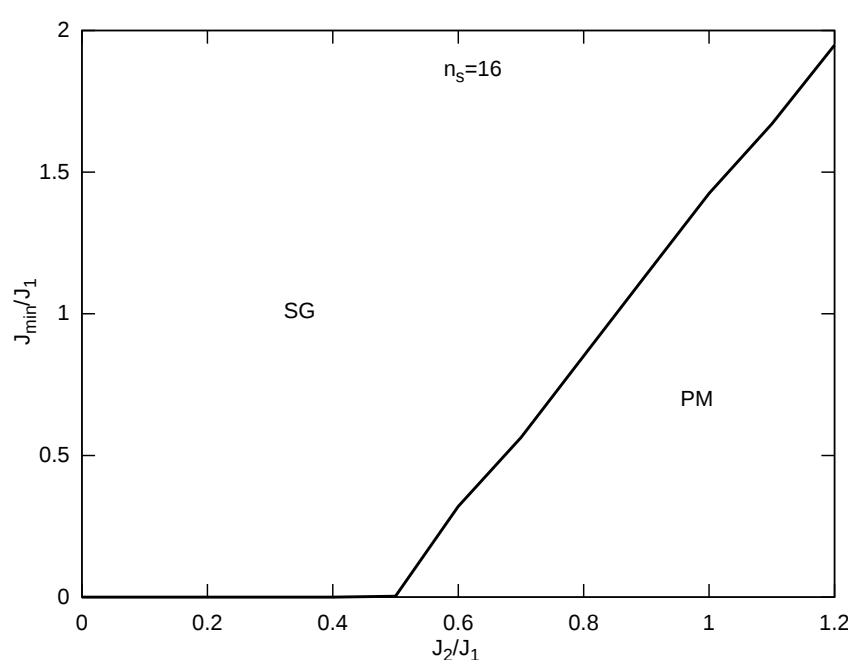

FIG. 6: Phase diagram $J_{\text {min }} / J_{1}$ versus $J_{2} / J_{1}$ for $n_{s}=16$ with $\mathrm{FE}\left(J_{1}\right)$ and $\mathrm{AF}\left(J_{2}\right)$ short-range intracluster interactions. The cluster shape is the same as Fig. (2-b).

\section{CONCLUDING REMARKS}

Summing up, we studied the interplay between intrinsic frustration and quenched disorder in a cluster spin glass model that considers intracluster interactions following the $J_{1}-J_{2}$ like model for finite clusters and longrange disordered intercluster interactions. The meanfield replica method is used to obtain an effective onecluster model, which is computed by exact diagonalization. The results make evident that the presence of AF clusters with intrinsic frustration is able to bring the SG order for lower disorder intensities. In other words, the intracluster frustration can increase the intensity of cluster magnetic moment favoring the intercluster SG order even in a weak disorder. Although the conclusions are obtained from a particular model, they indicate that the presence of small clusters in geometrically frustrated systems can help to stabilize the SG order within very small disordered regimes.
Acknowledgments

This work was partly supported by the Brazilian agencies CNPq, FAPERGS, CAPES and FAPERJ.
1 A. P. Ramirez, Annu. Rev. Mater. Sci. 24, 453 (1994).

2 Edited by C. Lacroix, P. Mendels, and F. Mila, Introduction to Frustrated Magnetism (Springer-Verlag, Heidelberg, 2011).

3 A. Andreanov, J. T. Chalker, T. E. Saunders, D. Sherrington, Phys. Rev. B 81, 014406 (2010)

4 J. J. Gardner, M. J. P Gingras, J. E. Greedan, Rev. Mod Phys. 82, 53 (2010).

${ }^{5}$ C.H. Booth, J.S. Gardner, G.H. Kwei, R. H. Heffner, F. Bridges, and M.A. Subramanian, Phys. Rev. B 62, R755 (2000).

6 W. Ratcliff II, S.-H. Lee, C. Broholm, S.-W. Cheong, and Q. Huang, Phys. Rev. B 65, 220406(R) (2002).

7 A.D. LaForge, S.H. Pulido, R.J. Cava, B.C. Chan, and A.P. Ramirez, Phys. Rev. Lett. 110, 017203 (2013).

8 D. Fioranit,J.L. Dormann, J.L. Tholence and J. L. Soubey- roux, J. Phys. C: Solid State Phys. 18, 3053 (1985)

${ }^{9}$ D. Sherrington, B W Southern, J. Phys. F: Metal Phys. 5, L49 (1975).

10 T.E. Saunders and J. T. Chalker, Phys. Rev. Lett. 98, 157201 (2007)

11 G. Parisi, J. Phys. A: Math. Gen. 13, 1101 (1980).

12 C. F. Silva, F. M. Zimmer, S. G. Magalhaes, C. Lacroix, Phys. Rev. E 86, 014406 (2012).

13 D.P. Landau and K. Binder, Phys. Rev. B 31, 5946 (1985).

14 J. L. Morn-Lpez, F. Aguilera-Granja, J. M. Sanchez, Phys. Rev. B 48, 3519 (1993).

15 A. Kalz and A. Honecker, Phys. Rev. B 84, 174407 (2011).

16 C. M. Soukoulis and K. Levin, Phys. Rev. B 18, 1439 (1978); C. M. Soukoulis, Phys. Rev. B 18, 3757 (1978). 